\title{
Tuning of observer-based estimators: theory and application to the on-line estimation of kinetic parameters
}

\author{
M. Perrier ${ }^{\mathrm{a}}$, S. Feyo de Azevedo ${ }^{\text {b }}$, E. C. Ferreira ${ }^{\mathrm{c}}$, D. Dochain ${ }^{\mathrm{d}, *}$ \\ ${ }^{a}$ Département de Génie Chimique, Ecole Polytechnique de Montréal Case Postale 6079, Succursale "Centre Ville”, Montréal H3C 3A7, Canada \\ ${ }^{\mathrm{b}}$ Departamento de Engenharia Química Faculdade de Engenharia da Universidade do Porto, Rua dos Bragas, 4099 Porto Cedex, Portugal \\ ${ }^{\mathrm{c}}$ Centro de Engenharia Biologica-IBQF, Universidade do Minho 4700 Braga, Portugal \\ ${ }^{\mathrm{d}}$ Senior Research Associate FNRS, Cesame, Université Catholique de Louvain Bâtiment Euler, av. G. Lemaître 4-6, 1348 Louvain-La-Neuve, Belgium
}

Received 29 October 1998; accepted 5 July 1999

\begin{abstract}
This paper deals with the tuning of observer-based estimators. Initially, these algorithms were designed for estimating on-line kinetic parameters, like specific growth rates, in bioprocesses, and have proved to be very successful in practical applications. Here a systematic tuning approach that allows a decoupled estimation of each parameter and the assignment of the estimator dynamics independently of the process dynamics is proposed. The presented approach is illustrated on an animal cell culture example in numerical simulation and with real-life data. (C) 2000 Elsevier Science Ltd. All rights reserved.
\end{abstract}

Keywords: Parameter estimation; State observer; Biotechnological process; Chemical process; Electrical system

\section{Introduction}

A key question in (bio)process control is how to monitor reactant and product concentrations and process parameters like reaction rates in a reliable and cost effective manner. However, it appears that in many practical applications, only some of the concentrations of the components involved that are critical for quality control are available for on-line measurement. For instance, dissolved oxygen concentration and gaseous flowrates are available for on-line measurement while the concentration values of biomass, substrates and/or synthesis products are often available via off-line analysis. An interesting alternative which circumvents and exploits the use of a model in conjunction with a limited set of measurements are Luenberger or Kalman observers. In these techniques, a model, which includes states that are measured as well as states that are not measured, is used in parallel with the process and the model states may then be used for feedback. This configuration may be used to reduce the effect of noise on measurements as well

\footnotetext{
*Corresponding author. Tel.: + 32-10-472-378; fax: + 32-10-472180.

E-mail address: dochain@auto.ucl.ac.be (D. Dochain).
}

as to reconstruct the states that are not measured. An introduction to these ideas can be found in e.g. Kwakernaak and Sivan (1972). These concepts were originally developed for linear problems. Because of the nonlinear characteristics of the bioprocess dynamics, it is of interest to extend these concepts and exploit particular structures for biochemical engineering application problems. Linearized versions (the linearized tangent model) of the process dynamics are computed from a Taylor's series expansion of a state space model around some equilibrium point and the observer theory referred to above can be applied. This idea is developed in Bastin and Dochain (1990). These modified observers, particularly the extended Kalman filter, have found applications in some (bio)chemical processes (e.g. Stephanopoulos \& San, 1984; Tsobanakis, Lee, Phillips \& Georgakis, 1992; Caminal, Lafuente, Lopez-Santin, Poch \& Sola, 1987, Wells, 1971; MacGregor, Kojub, Penlidis \& Hamielec, 1986; Kiparissides, MacGregor \& Hamielec, 1981).

One of the reasons for the popularity of the extended Kalman filter is that it is easy to implement since the algorithm can be derived directly from the state space model. However, since (as is the extended Luenberger observer) it is based on a linearized model of the process, the stability and convergence properties are essentially local and valid around an equilibrium point, and it is 
rather difficult to guarantee its stability over wide ranges of operation. Ljung (1979) shows that the extended Kalman filter for state and parameter estimation of linear systems may give biased estimates or even diverge if it is not carefully initialized. It must also be pointed out that the derivation of the extended Kalman filter is based on some stochastic assumptions on the measurement and process noises, which might be questionable in practice.

One reason for the problem of convergence of extended Kalman filter is that, in order to guarantee the (arbitrarily chosen) exponential convergence of the observer, the process must be locally observable, i.e. the linearized tangent model must be observable and fulfill the classical observability rank condition. This condition, as it turns out, is restrictive in many practical situations and may account for the failure of extended Kalman filter to find widespread application (e.g. Bastin \& Dochain, 1990; Bastin \& Levine, 1990; Dochain \& Chen, 1992).

Another problem is that the theory for the extended Luenberger and Kalman observers is developed using a perfect knowledge of the system parameters, in particular of the process kinetics: it is difficult to develop error bounds and there is often a large uncertainty on these parameters.

It appears from the above remarks that there is a clear incentive to develop new methodologies for the on-line estimation of the unmeasured concentration variables in biochemical reaction systems that do not rely on the explicit use of kinetic models. The design of monitoring tools for the on-line estimation of process variables and parameters has been quite an active research area over the past 20 years (see e.g. Bastin \& Dochain, 1986; Dochain, Perrier \& Ydstie, 1992; Doyle III, 1997; Goodwin, Mc Innis \& Long, 1980). Alternative approaches have been proposed to extended Kalman filter that use process physics in a more direct manner to develop nonlinear observers applicable to the estimation problem of (bio)chemical reactors. The proposed observers are based on the well-known nonlinear model of the process without the knowledge of the process kinetics being necessary. For state estimation, these observers have been called asymptotic observers; for parameter estimation, observer-based estimators have been developed in particular; these are the objects of the present paper.

The development of new state and parameter estimators in order to palliate the deficiencies of classical observers encountered in areas like process control is a very active reserach area. Other options like neural networks based estimators (e.g. Cannon \& Slotine, 1995) and $H_{\infty}$ based estimators (e.g. Reza Moheimani, Satkin \& Petersen, 1996) have been proposed in the literature. Compared to these approaches, the observer-based estimator presents the following specific characteristics: it does not need to introduce a black-box (nonlinear) modelling of the uncertain parameters, and the stability analysis does not lead to conservative tuning rules (as may be the case with $H_{\infty}$ based algorithms).

The observer-based estimator has initially been designed to estimate on-line specific growth rates in bioprocesses (Bastin \& Dochain, 1986), then extended to the on-line estimation of kinetic parameters (Bastin \& Dochain, 1990). It proved to be very successful in a large variety of bioprocess applications in the fields of food processes, pharmaceutical processes and environmental processes (e.g. Atroune, Cheruy, Flandrois \& Carret, 1988; Bastin \& Dochain, 1990; Bourrel, Dochain, Babary \& Queinnec, 1999; Claes \& Van Impe, 1998; Oliveira, Ferreira, Oliveira \& Feyo de Azevedo, 1996; Flaus, Pons, Cheruy \& Engasser, 1989; Pomerleau, Perrier \& Bourque, 1995; Pomerleau \& Viel, 1992; Sulmon, 1997). If the stability properties of the estimation algorithm are well understood (Bastin \& Dochain, 1990), the tuning of such estimators aimed at tracking parameters that are typically time-varying deserves particular attention: this matter has already been studied in (Oliveira, Ferreira, Oliveira, \& Feyo de Azevedo, 1996) and (Pomerleau \& Perrier, 1990), which propose a calibration strategy of the estimator design parameters independent of the values of the state variables. In this paper, it is proposed to further develop the tuning approach. This is achieved via two steps: a state transformation, and the re-arrangement of the estimator state vector entries. At this point the main objective of the present paper is to systematize the tuning approach. The basic stability and convergence properties of the estimator shall only be recalled briefly. Finally the observer-based estimator is designed for a larger class of dynamical systems.

The paper is organized as follows. Section 2 is concerned with the design of the observer-based estimator. Section 3 deals with the derivation of the tuning rules. And in Section 4, the tuning of the estimation algorithm is illustrated on an animal cell culture example via numerical simulations and real-life data.

\section{Design of the observer-based estimator}

\subsection{Dynamical system equations}

Consider a system whose dynamics are described by the following equations:

$\frac{\mathrm{d} x}{\mathrm{~d} t}=F_{1}(x) \theta+F_{2}(x)$,

where $x$ is the state vector $(\operatorname{dim}(x)=n), \theta$ is the vector of (unknown) parameters $\left(\operatorname{dim}(\theta)=p\right.$ ), and $F_{1}(x)$ and $F_{2}(x)$ are (matrix), generally nonlinear, functions of the state vector $x$. A typical example is the mass and energy 


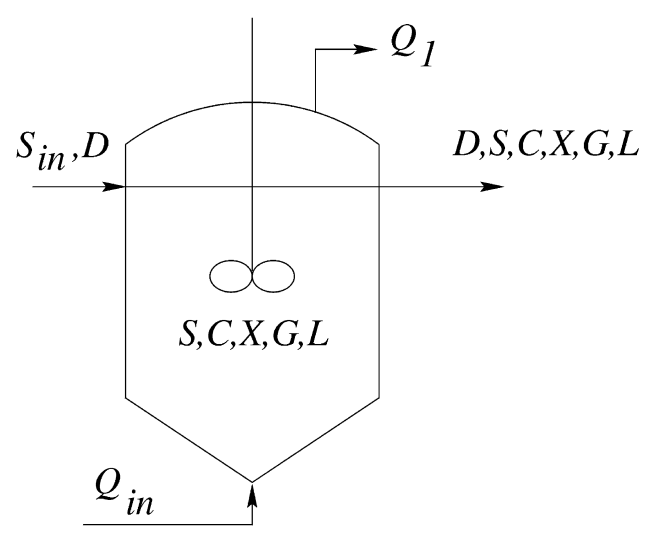

Fig. 1. Schematic view of the animal cell culture stirred tank reactor.

balance equations of reaction systems, including chemical and biochemical stirred tank reactors (STRs) (Fig. 1) (see Bastin \& Dochain, 1990; Dochain, 1994):

$\frac{\mathrm{d} x}{\mathrm{~d} t}=K G(x) \theta+F-Q-D x$,

where $x, K, G(x) \theta, F, Q$, and $D$ are the vector of process component concentrations $(\mathrm{g} / \mathrm{l})$ (plus the temperature $(\mathrm{K})$ in case of energy balance), the stoichiometric or yield coefficient matrix, the reaction rate vector $(\mathrm{g} / \mathrm{l} / \mathrm{h})$, the feedrate vector $(\mathrm{g} / \mathrm{l} / \mathrm{h})$, the gaseous outflow rate vector $(\mathrm{g} / \mathrm{l} / \mathrm{h})$, and the dilution rate $\left(\mathrm{h}^{-1}\right)$ (which is more precisely the ratio of the influent flow rate over the reaction medium volume), respectively. The mass and energy balance dynamic model given above is indeed composed of two terms: transport dynamics $(F-Q-D x)$ due to the flow of matter through the reactor, and conversion $(K G(x) \theta)$ due to the (bio)chemical transformations.

Other dynamical systems with structure given by (1) can be found, e.g. in electrical systems (e.g. Ortega \& Espinosa, 1993; Mbihi, Dochain \& Turgeon, 1993).

\subsection{Example: animal cell culture}

Throughout the paper the following bioprocess example shall be used as a guiding thread: an animal cell culture. The choice of this example from the numerous examples available in the literature in general, and from the authors' practical experience in particular, is obviously arbitrary.

The animal cell culture considered here is a human embryo kidney (HEK-293) cell culture (Siegwart et al., 1999). It has been shown in Siegwart et al. (1999) that the process is characterized by the following reaction network:

$k_{1} S+k_{2} C \rightarrow X+k_{3} G$,

$k_{4} S \rightarrow X+k_{5} L$, where $S, C, X, G$ and $L$ represent the glucose, dissolved oxygen, yeast, carbon dioxide and lactate, respectively. Reactions (3) and (4) are an oxidation (respiration) reaction on glucose, and a glycolysis (fermentation) on glucose, respectively. The dynamics of the process in a stirred tank reactor (Fig. 1) are given in the matrix format (2) by the following vectors and matrices:

$x=\left[\begin{array}{c}S \\ C \\ X \\ G \\ L\end{array}\right], \quad K=\left[\begin{array}{cc}-k_{1} & -k_{4} \\ -k_{2} & 0 \\ 1 & 1 \\ k_{3} & 0 \\ 0 & k_{5}\end{array}\right]$,

$G(x) \theta=\left[\begin{array}{l}\mu_{R} X \\ \mu_{F} X\end{array}\right]$,

$F=\left[\begin{array}{c}D S_{i n} \\ Q_{i n} \\ 0 \\ 0 \\ 0\end{array}\right], \quad Q=\left[\begin{array}{c}0 \\ 0 \\ 0 \\ Q_{1} \\ 0\end{array}\right]$,

$k_{j}(j=1, \ldots, 5)$ are yield coefficients, $S_{i n}$ is the influent glucose concentration $(\mathrm{g} / \mathrm{l}), Q_{i n}$ is the oxygen feedrate $(\mathrm{g} / \mathrm{l} / \mathrm{h}), Q_{1}$ is the $\mathrm{CO}_{2}$ outflow rate $(\mathrm{g} / \mathrm{l} / \mathrm{h})$, and $\mu_{i}$ $(i=R, F)$ are the specific growth rates $(1 / \mathrm{h})$ associated to each growth reaction.

The different possibilities for distributing terms in $G(x)$ and $\theta$ are now briefly discussed. The above model formulation suggests the following distribution (this is the one considered below):

$G(x)=\left[\begin{array}{ll}X & 0 \\ 0 & X\end{array}\right], \quad \theta=\left[\begin{array}{l}\mu_{R} \\ \mu_{F}\end{array}\right]$.

Many other options are possible, depending on the level of knowledge and uncertainty one has in the process kinetics. As a matter of illustration, one may consider:

1. That the whole reaction rate vector is unknown:

$$
G(x)=I, \quad \theta=\left[\begin{array}{l}
\mu_{R} X \\
\mu_{F} X
\end{array}\right] .
$$

2. Or that, in accordance with kinetics laws, the reaction rates are explicitely related to the limiting substrates (the simplest way to express this is to write: $\mu_{R}=\rho_{1} S C$, and $\mu_{F}=\rho_{2} S$ ), and then to assume that the parameters $\rho_{i}$ are unknown; then

$$
G(x)=\left[\begin{array}{cc}
S C X & 0 \\
0 & S X
\end{array}\right], \quad \theta=\left[\begin{array}{l}
\rho_{1} \\
\rho_{2}
\end{array}\right] .
$$


3. Or that one has some knowledge of the kinetics model structure (e.g. the Monod model), and that the maximum specific growth rates $\mu_{\max , i}(i=1,2)$ are unknown while the affinity constants $K_{S 1}, K_{S 2}, K_{C 1}$ are known; this leads to the following definitions for $G(x)$ and $\theta$ :

$$
\begin{aligned}
& G(x)=\left[\begin{array}{cc}
\frac{S C X}{\left(K_{S 1}+S\right)\left(K_{C 1}+C\right)} & 0 \\
0 & \frac{S X}{K_{S 2}+S}
\end{array}\right], \\
& \theta=\left[\begin{array}{l}
\mu_{\max , 1} \\
\mu_{\max , 2}
\end{array}\right] .
\end{aligned}
$$

\subsection{Observer-based estimator}

Assume that:

H1. the $p$ parameters $\theta$ are unknown and possibly timevarying (with bounded time variations $\|\mathrm{d} \theta / \mathrm{d} t\|$ $<M$ );

H2. $p$ state variables are available for on-line measurement.

From assumption $\mathrm{H} 2$, a state partition can be defined

$$
x=\left[\begin{array}{l}
x_{1} \\
x_{2}
\end{array}\right]
$$

$x_{1}$ being the measured variables, and $x_{2}$ the unmeasured ones. The dynamical equations can then be rewritten as follows:

$$
\begin{aligned}
& \frac{\mathrm{d} x_{1}}{\mathrm{~d} t}=F_{11}(x) \theta+F_{21}(x), \\
& \frac{\mathrm{d} x_{2}}{\mathrm{~d} t}=F_{12}(x) \theta+F_{22}(x) .
\end{aligned}
$$

Further assume that:

H3. $F_{11}$ can be written as the product of two $p \times p$ matrices: $F_{11}=F_{3}(x) F_{4}(x)$ with $F_{4}$ a diagonal matrix $\left(F_{4}=\operatorname{diag}\left\{f_{4, i}\right\}, i=1\right.$ to $\left.p\right)$ and $F_{3}$ being full rank for all admissible values of $x$ (for (bio)chemical processes for instance, only positive values of the state variables (i.e. concentrations and possibly temperature) are considered);

H4. $F_{11}(x)$ and $F_{21}(x)$ are known functions of $x$.

Under assumption H3, it may be possible to build an open-loop observer to reconstruct the time evolution of $x_{2}$ independently of the unknown parameters $\theta$. In the particular example of reaction systems, such an observer has asymptotically stable dynamics as long as the dilution rate $D$ is persistently exciting (i.e., here, not equal to zero for too long), and is called an asymptotic observer (see Bastin \& Dochain, 1990). Another example of such open loop observers can be found in Ortega and Espinosa (1993) for induction motors. In the following, we consider that states included in $x_{2}$ are either accessible for on-line measurement or available via such observers, or that the dynamics of $x_{1}$ are independent of $x_{2}$.

In the context of stirred tank reactors (STRs),

$F_{3}=K_{1}, \quad F_{4}=G(x)$

with $K_{1}$ the yield coefficient matrix associated to $x_{1}$. Note that by constructing and assuming $\mathrm{H} 2$, the matrix $G(x)$ is a $p \times p$ matrix. Assumption $\mathrm{H} 4$ then means that the feedrates $F_{1}$ (associated to $x_{1}$ ), the gaseous outflow rates $Q_{1}$ (associated to $x_{1}$ ), the dilution rate $D$ are known (via on-line measurements or user's choice), as well as the stoichiometric (yield) coefficients in $K_{1}$ and the function $G(x)$.

The design of the observer-based estimator is based on (12) and follows indeed the line of reasoning for the design of Luenberger observers. This gives the following estimator equations:

$\frac{\mathrm{d} \hat{x}_{1}}{\mathrm{~d} t}=F_{11}(x) \hat{\theta}+F_{21}(x)-\Omega\left(x_{1}-\hat{x}_{1}\right)$,

$\frac{\mathrm{d} \hat{\theta}}{\mathrm{d} t}=\left[F_{11}(x)\right]^{\mathrm{T}} \Gamma\left(x_{1}-\hat{x}_{1}\right)$.

The basic motivation of the above structure for the estimator is the following. Like in a classical observer, the estimator equations are the combination of the process model $\left(F_{11}(x) \hat{\theta}+F_{21}(x)\right)$ and correction terms $\left(-\Omega\left(x_{1}-\hat{x}_{1}\right)\right.$ and $\left.\left[F_{11}(x)\right]^{\mathrm{T}} \Gamma\left(x_{1}-\hat{x}_{1}\right)\right)$ on the measured variables. In the above observer-based estimator, the parameters $\theta$ are assimilated as states without dynamics. The weighting factor $\left[F_{11}(x)\right]^{\mathrm{T}}$ in (16) is indeed the term multiplying the unknown parameter in the model equation: its introduction in the estimator equation derives from classical estimator design (e.g. Goodwin \& Payne, 1977) and it is usually called the regressor.

\subsection{Example: animal cell culture (continued)}

Assume that the glucose concentration $S$ and the lactate concentration $L$ are accessible for on-line measurements, and that the objective is to estimate on-line the specific growth rates. Then the vectors and matrices of the observer-based estimator specializes as follows:

$$
\begin{aligned}
& x_{1}=\left[\begin{array}{l}
S \\
L
\end{array}\right], \quad F_{11}=K_{1} X=\left[\begin{array}{cc}
-k_{1} X & -k_{4} X \\
0 & k_{5} X
\end{array}\right], \\
& \theta=\left[\begin{array}{l}
\mu_{R} \\
\mu_{F}
\end{array}\right] \\
& F_{21}=F_{1}-Q_{1}-D x_{1}=\left[\begin{array}{c}
D S_{i n} \\
0
\end{array}\right]-D x_{1} .
\end{aligned}
$$




\subsection{A comparison with the extended Kalman filter}

Before going further, it may be useful to gain an insight about the observer-based estimator by comparing it with the extended Kalman filter (extended Kalman filter), at least in a simple bioprocess example. Consider a simple microbial growth process in a stirred tank reactor (STR). The dynamic mass balances for biomass and substrate are expressed by the following equations:

$\frac{\mathrm{d} X}{\mathrm{~d} t}=\mu X-D X$

$\frac{\mathrm{d} S}{\mathrm{~d} t}=-k_{1} \mu X+D S_{\text {in }}-D S$.

Assume that the biomass concentration $X$ is available for on-line measurement and that the objective is to estimate on-line the specific growth rate $\mu$. The observer-based estimator $(\mathrm{OBE})$ is given by the following equations:

$\frac{\mathrm{d} \hat{X}}{\mathrm{~d} t}=\hat{\mu} X-D X+\omega_{1}(X-\hat{X})$,

$\frac{\mathrm{d} \hat{\mu}}{\mathrm{d} t}=\gamma_{1} X(X-\hat{X})$

and the extended Kalman filter) takes the following form (see Fig. 2):

$\frac{\mathrm{d} \hat{X}}{\mathrm{~d} t}=\hat{\mu} \hat{X}-D \hat{X}+r_{11}(X-\hat{X})$,

$\frac{\mathrm{d} \hat{\mu}}{\mathrm{d} t}=r_{22}(X-\hat{X})$,

$\frac{\mathrm{d} r_{11}}{\mathrm{~d} t}=2(\hat{\mu}-D) r_{11}+2 \hat{X} r_{12}-r_{11}^{2}$,

$\frac{\mathrm{d} r_{12}}{\mathrm{~d} t}=(\hat{\mu}-D) r_{12}+\hat{X} r_{22}-r_{11} r_{12}$,

$\frac{\mathrm{d} r_{22}}{\mathrm{~d} t}=-r_{12}^{2}+\sigma$,

where $\sigma$ is the weighting factor of the extended Kalman filter quadratic criterion and $r_{11}, r_{12}, r_{22}$ are the entries of the gain matrix $R$ (see e.g. Bastin \& Dochain, 1990; Kwakernaak \& Sivan, 1972) for further details). First, note the extended Kalman filter is more complex and is required to fix four parameters $\left(\sigma, r_{11}(0), r_{12}(0), r_{22}(0)\right)$. The performance of both observers is illustrated when $\mu$ is constant (steady state) and under the following conditions:

$\mu=\frac{\mu_{\max } S}{K_{S}+S}, \quad \mu_{\max }=0.33 \mathrm{~h}^{-1}, \quad K_{S}=5 \mathrm{~g} / 1$,

$D=0.05 \mathrm{~h}^{-1}, \quad k_{1}=2, \quad S_{\text {in }}=5 \mathrm{~g} / 1$,
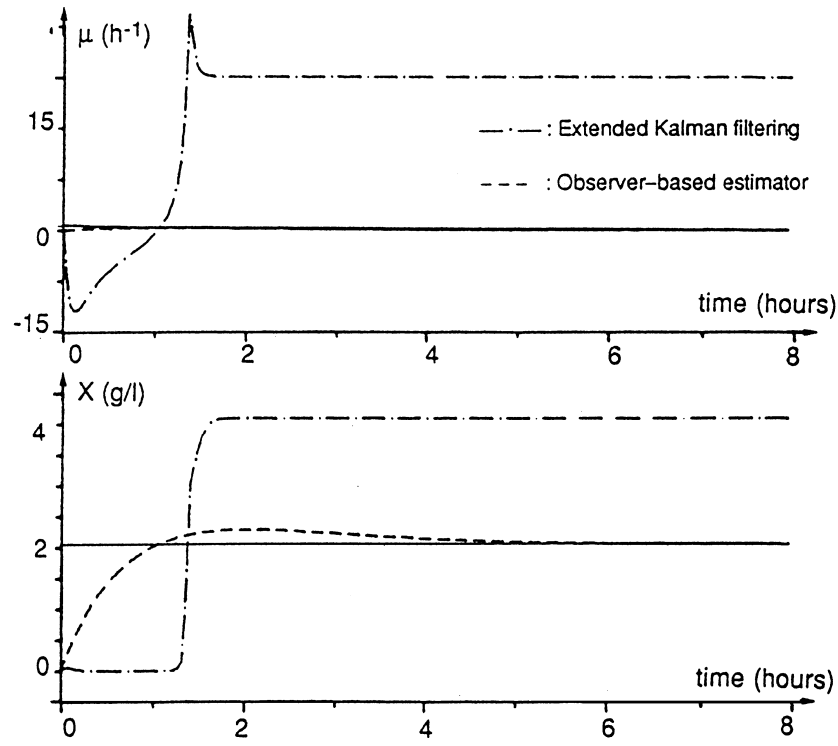

Fig. 2. Comparison with the extended Kalman filter.

$$
\begin{aligned}
& X(0)=2.05 \mathrm{~g} / 1, \quad S(0)=0.89 \mathrm{~g} / 1, \\
& \hat{\mu}(0)=0, \quad \hat{X}(0)=0, \quad \omega_{1}=0.5, \quad \gamma_{1}=0.24 \\
& \sigma=0.01, \quad r_{11}(0)=1, \quad r_{12}(0)=-99, \quad r_{22}(0)=10000 .
\end{aligned}
$$

The initial value of the gain matrix $R$ is chosen so as to be a positive-definitive matrix. Note that the observer-based estimator converges (as expected from the theoretical stability and convergence properties of the estimator, see Bastin \& Dochain, 1990) while the extended Kalman filter tends to fix $\hat{\mu}$ to a value close to $22 \mathrm{~h}^{-1}$ (i.e. more than two orders of magnitude away from the correct value $\left(0.05 \mathrm{~h}^{-1}\right)$. Even if in this simple example it would not be difficult to set the extended Kalman filter design parameters to values that will give a good estimate of $\mu$, it illustrates the potential difficulty to calibrate properly the extended Kalman filter in more complex situations, and its failure to find widespread application in (bio)chemical processes.

\section{Tuning of the observer-based estimator: theory}

The theoretical stability analysis of the above observer-based estimator is available in Bastin and Dochain (1990): the main requirements are the negative definiteness of $\Omega^{\mathrm{T}} \Gamma+\Gamma \Omega$ and the persistence of excitation of $F_{11}(x)$. However, its tuning may be difficult and intricate in practice, because of the close interaction of the unknown parameters in the estimator equations, and because its dynamics depends on the process variables. The latter may be of minor importance if the system is 
operated around a steady state, but it will become crucial if the system covers a large range of operating conditions (as for fed-batch and batch reactors, or process start-ups and grade changes) possibly with large variations of the state variables, and as a consequence of the matrix $F_{11}(x)$. Good tracking capabilities of the parameters' variations are particularly essential in these circumstances. However, in the above form (15), (16) of the observer-based estimator, tuning may give very conservative values for the design parameters $\Gamma$ and $\Omega$, and result in bad tracking performance in some of the operating regions.

The above objectives for the tuning of the observerbased estimator can be achieved by considering first the following two steps in the reformulation of the algorithm:

1. a state transformation;

2. the re-arrangement of the estimator state vector entries.

\subsection{A state transformation}

Consider the following state transformation:

$z=F_{3}^{-1} x$.

Then the dynamic equations of the system can be rewritten as follows:

$\frac{\mathrm{d} z}{\mathrm{~d} t}=F_{4}(x) \theta+F_{3}^{-1}(x) F_{21}(x)$.

Due to the above transformation, only one state variable is associated with each unknown parameter $\theta$. In the specific case of stirred tank reactors, the invertibility of $F_{3}\left(=K_{1}\right)$ results from the independence of the $p$ reactions and of the $p$ measured variables (see Bastin $\&$ Dochain, 1990). The above transformation has been already proposed in Pomerleau and Perrier (1990) and Oliveira et al. (1996).

The observer-based estimator can now be re-designed on the basis of Eq. (33) :

$\frac{\mathrm{d} \hat{z}}{\mathrm{~d} t}=F_{4}(x) \hat{\theta}+F_{3}^{-1}(x) F_{21}(x)-\Omega(z-\hat{z})$,

$\frac{\mathrm{d} \hat{\theta}}{\mathrm{d} t}=\Gamma(z-\hat{z})$.

Due to the transformation (32), the observer-based estimator is now reformulated in a decoupled format for the unknown parameters $\theta_{i}(i=1, \ldots, p)$. Because of the decoupled estimation formulation, an obvious choice for the matrices $\Omega$ and $\Gamma$ are diagonal matrices:

$$
\begin{gathered}
\Omega=\operatorname{diag}\left\{-\omega_{i}\right\}, \quad \Gamma=\operatorname{diag}\left\{\gamma_{i}\right\}, \quad \omega_{i}>0, \quad \gamma_{i}>0, \\
\quad i=1, \ldots, p .
\end{gathered}
$$

In the above formulation of the estimation scheme, we have removed the regressor term $F_{4}(x)(G(x)$ in the STR example) from the estimation equation of $\theta$ (35) (see (50)): since one of its main role is to explicitely transfer the coupling between the unknown parameters and the measured variables, its presence is not anymore essential.

\subsection{Re-arrangement of the estimator state vector entries}

The final step before the formulation of the tuning rule consists of a re-arrangement of the estimator's equations. Let us gather each variable $z_{i}$ with its related parameter $\theta_{i}$ and re-arrange the entries of the vector $[z, \theta]^{\mathrm{T}}$ in the following order in a vector $\zeta$ :

$\zeta=\left[\begin{array}{c}z_{1} \\ \theta_{1} \\ z_{2} \\ \theta_{2} \\ \cdots \\ z_{p} \\ \theta_{p}\end{array}\right]$

\subsection{Basic tuning rule}

Let us first define the estimation error:

$e=\zeta-\hat{\zeta}$

The estimation error dynamics are readily derived from Eqs. (33)-(35):

$\frac{\mathrm{d} e}{\mathrm{~d} t}=A e+b$

with a block diagonal matrix $A$ with $2 \times 2$ blocks:

$A=\operatorname{diag}\left\{A_{i}\right\}, \quad A_{i}=\left[\begin{array}{ll}-\omega_{i} & f_{4, i}(x) \\ -\gamma_{i} & 0\end{array}\right], \quad i=1, \ldots, p$

and $b$ equal to:

$$
b=\left[\begin{array}{c}
0 \\
\frac{\mathrm{d} \theta_{1}}{\mathrm{~d} t} \\
0 \\
\frac{\mathrm{d} \theta_{2}}{\mathrm{~d} t} \\
\cdots \\
0 \\
\frac{\mathrm{d} \theta_{p}}{\mathrm{~d} t}
\end{array}\right] .
$$


The characteristic equation of the matrix $A, \operatorname{det}(\lambda I-A)$, is equal to

$\operatorname{det}(\lambda I-A)=\prod_{i=1}^{p}\left(\lambda^{2}+\omega_{i} \lambda+\gamma_{i} f_{4, i}(x)\right)$.

The key idea of the tuning rule consists of choosing each $\gamma_{i}$ inversely proportional to the corresponding term $f_{4, i}(x)$ :

$\gamma_{i}=\frac{\bar{\gamma}_{i}}{f_{4, i}(x)}, \quad \bar{\gamma}_{i}>0, \quad i=1, \ldots, p$.

With the choice above, the characteristic equation (42) is rewritten as follows:

$\operatorname{det}(\lambda I-A)=\prod_{i=1}^{p}\left(\lambda^{2}+\omega_{i} \lambda+\bar{\gamma}_{i}\right)$

and the observer-based estimator dynamics are now independent of the state variables. Such a choice corresponds to a Lyapunov transformation (see Perrier $\&$ Dochain, 1993). It is obviously valid for values of $f_{4, i}(x) \neq 0$ : this condition is usually met easily in (bio)process applications, as will be illustrated in the following section.

The values of the design parameters can then be set to arbitrarily fix the estimator's dynamics for each unknown parameter $\theta_{i}$. Since the estimator reduces via the transformations to a set of independent second-order linear systems, the classical rules for assigning the dynamics of second-order linear systems apply straighforwardly here. The reader is therefore referred to the classical automatic control textbooks for further information on the subject. However, the following basic guidelines are suggested.

One important guideline is to choose real poles:

$\omega_{i}^{2}-4 \bar{\gamma}_{i} \geqslant 0$.

The objective is then to avoid inducing oscillations in the estimation of the parameters that do not correspond to any physical phenomenon related to the estimated reaction rates.

Pomerleau and Perrier (1990) suggest choosing double poles, i.e.

$\bar{\gamma}_{i}=\frac{\omega_{i}^{2}}{4}$.

Oliveira et al. (1996) propose as an alternative to choose complex poles with a damping factor equal to 0.7 in order to increase the speed of convergence of the estimator with a reduced overshoot. (Generally speaking, the damping factor can be freely chosen; the choice may then depend on the type and nature of the application, of the time variations of the parameter to be estimated, and of the noise on the measured data. This means that there are two design parameters per estimated parameters.)

Then the tuning of the estimation algorithm reduces to the choice of one design parameter, $\omega_{i}$, per estimated parameter. This allows to have a design procedure that has the double advantage of being simple (one design parameter) and flexible (each parameter estimation can be tuned differently if needed, e.g. if the time variations of the parameters are different).

So far, we have suggested that it is possible to assign arbitrarily the dynamics of the estimator. However in the presence of noisy data, it appears that indeed a compromise has to be made between a fast estimator convergence and a good noise rejection. A detailed and somewhat involved study is performed in Bastin and Dochain (1990) (pp. 162-172) to analyze the performance of the observer-based estimator both in theory and in numerical simulation in the presence of bounded noisy data in the particular case of the estimation of the specific growth rate of a simple microbial growth process (already mentioned in Section 2.5). The theoretical optimization analysis is based on the evaluation of the asymptotic properties of the estimator and results in the following optimal value for $\omega_{1}$ :

$\omega_{1, o p t}=2 \sqrt{\frac{k_{1} M_{1}}{\alpha\left(k_{1} M_{2}^{2}+S_{\max } M_{2}\right)}}, \quad 0<\alpha<1$,

where $M_{1}$ and $M_{2}$ are the upper bounds on the time derivative of $\mu$ and on the measurement noise, respectively, and $S_{\max }$ the maximum value of the influent substrate concentration $S_{i n}$. This result is probably rather conservative because it is based on upper bounds for the measurement noise, the time variation of $\mu$ and the influent substrate concentration, but it is qualitatively confirmed by numerical simulation studies which also give a value of the design parameters that minimizes the estimation error. Because it is conservative, the theoretical optimum has to be interpreted with care, but since it is qualitatively correct, our suggestion (also based on our practical experience), in the presence of noisy measurement, is to perform numerical simulations with plausible reaction rate model and noise in order to get a first initial guess for the design parameter values which can then be adjusted when applied to the real process.

\section{Tuning of the observer-based estimator: application to animal cell culture}

For stirred tank reactors, transformation (32) specializes as follows:

$z=K_{1}^{-1} x$ 
and the observer-based estimator equations then become

$\frac{\mathrm{d} \hat{z}}{\mathrm{~d} t}=G(x) \hat{\theta}+K^{-1}(F-Q)-D z-\Omega(z-\hat{z})$,

$\frac{\mathrm{d} \hat{\theta}}{\mathrm{d} t}=\Gamma(z-\hat{z})$

In the animal cell culture example, it is written as follows:

$z=\left[\begin{array}{l}z_{1} \\ z_{2}\end{array}\right]=\left[\begin{array}{c}-\frac{S}{k_{1}}-\frac{k_{4}}{k_{5}} L \\ \frac{k_{1} k_{5}}{k_{5}}\end{array}\right]$.

This leads to the following formulation of the observerbased estimator:

$\frac{\mathrm{d} \hat{z}_{1}}{\mathrm{~d} t}=\hat{\mu}_{R} X-D z_{1}-\frac{1}{k_{1}} D S_{i n}+\omega_{1}\left(z_{1}-\hat{z}_{1}\right)$,

$\frac{\mathrm{d} \hat{\mu}_{R}}{\mathrm{~d} t}=\gamma_{1}\left(z_{1}-\hat{z}_{1}\right)$,

$\frac{\mathrm{d} \hat{z}_{2}}{\mathrm{~d} t}=\hat{\mu}_{F} X-D z_{2}+\omega_{2}\left(z_{2}-\hat{z}_{2}\right)$

$\frac{\mathrm{d} \hat{\mu}_{F}}{\mathrm{~d} t}=\gamma_{2}\left(z_{2}-\hat{z}_{2}\right)$.

\subsection{Numerical simulation}

In order to illustrate the performance of the observerbased estimator and of the proposed tuning rule, numerical simulation results are shown. These have been performed on the basis of a dynamical model of the animal cell culture presented in Section 2.2. The process is run in fedbatch mode, i.e. with a variable volume $V$, given by the following mass balance equation:

$\frac{\mathrm{d} V}{\mathrm{~d} t}=F_{\text {in }}$

where $F_{\text {in }}$ is the influent flow rate, related to the dilution rate as follows:

$D=F_{\text {in }} / V$.

The simulation model requires kinetic models for both specific growth rates; these have been found experimentally to have qualitatively the following structures (see Siegwart et al., 1999):

$\mu_{R}=\mu_{\max , 1} \frac{S}{K_{R}+S} \frac{K_{L}}{K_{L}+L}$,

$\mu_{F}=\mu_{\max , 2} \frac{S}{K_{F}+S}$, i.e. a Monod structure, plus inhibition of respiration by the lactate. The parameters of the model are equal to (see Siegwart et al., 1999):

$\mu_{\max , 1}=0.055 \mathrm{~h}^{-1}, \quad K_{R}=10 \mathrm{mM}$,

$K_{L}=50 \mathrm{mM}$,

$\mu_{\max , 2}=0.045 \mathrm{~h}^{-1}, \quad K_{F}=10 \mathrm{mM}$,

$k_{1}=1.7, \quad k_{4}=8.5, \quad k_{5}=17$.

The variables are initialized at the following values:

$X(0)=0.1810^{6}$ cells $/ \mathrm{ml}, \quad S(0)=21 \mathrm{mM}$,

$L(0)=0.13 \mathrm{mM}$,

$V(0)=19 \mathrm{~L}, \quad V_{f}=19.05 \mathrm{~L}, F=0.5 \mathrm{~mL} / \mathrm{h}$,

$S_{\text {in }}=3.3 \mathrm{M}$.

The time duration of the simulated operation is $110 \mathrm{~h}$, i.e. $100 \mathrm{~h}$ with a constant flow rate, and $10 \mathrm{~h}$ batch. The value of the biomass concentration $X$ is given via an asymptotic observer (Bastin \& Dochain, 1990), which takes here the following form:

$\frac{\mathrm{d} Z}{\mathrm{~d} t}=-D Z+\frac{1}{k_{1}} D S_{i n}$,

$\hat{X}=Z-\frac{S}{k_{1}}-\frac{k_{4}-k_{1}}{k_{1} k_{5}} L$.

The initial estimated specific growth rates have been set to the following values:

$\hat{\mu}_{R}(0)=0 \mathrm{~h}^{-1}, \quad \hat{\mu}_{F}(0)=0 \mathrm{~h}^{-1}$.

In the numerical simulations, $Z$ has been initialized by considering $20 \%$ error on the initial value of the biomass, i.e. $Z(0)=1.2 X(0)+S(0) / k_{1}+\left(k_{4}-k_{1}\right) L(0) / k_{1} / k_{5}$.

The organization of the figures is similar in the Figs. 3 , 5 and 6: Figs. a and $\mathrm{b}$ present the concentration of glucose and lactate, Figs. $c$ and $d$ the estimation results of the specific growth rates $\mu_{R}$ and $\mu_{F}$, Fig. e presents the biomass concentration (simulated value in Figs. $3 \mathrm{e}$ and 5e, off-line data in Fig. 6e, compared to the asymptotic observer estimation; Fig. 5e presents also the observerbased estimation validation, see below), and Fig. f gives the volume.

Fig. 3 shows the convergence for different values of $\omega_{1}$ and $\omega_{2}\left(1 \mathrm{~h}^{-1}, 10 \mathrm{~h}^{-1}\right)$ : note that as expected the convergence speed increases as $\omega_{i}$ does. Fig. 4 illustrates the advantage of the estimator design (43) developed here over the classical one (16). The same values of the design parameters have been considered: $\omega_{i}=10, \gamma_{i}=\bar{\gamma}_{i}=25(i=1,2)$. Note that if the performance of both estimator designs are similar in the second part, the performance with the classical design is worse than the pole-placement design in the first part. Fig. 5 

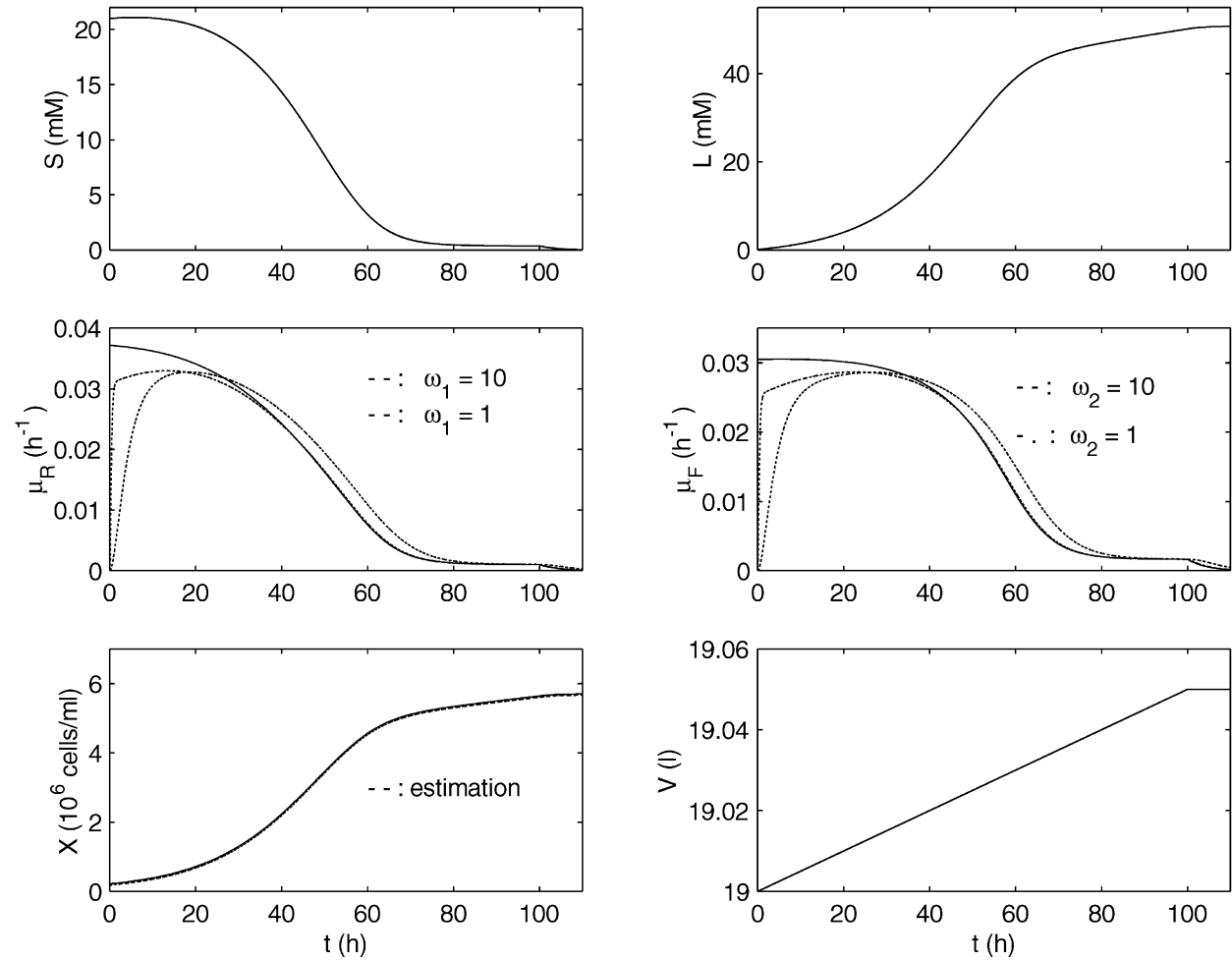

Fig. 3. Numerical simulation of the observer-based estimator with different gains.
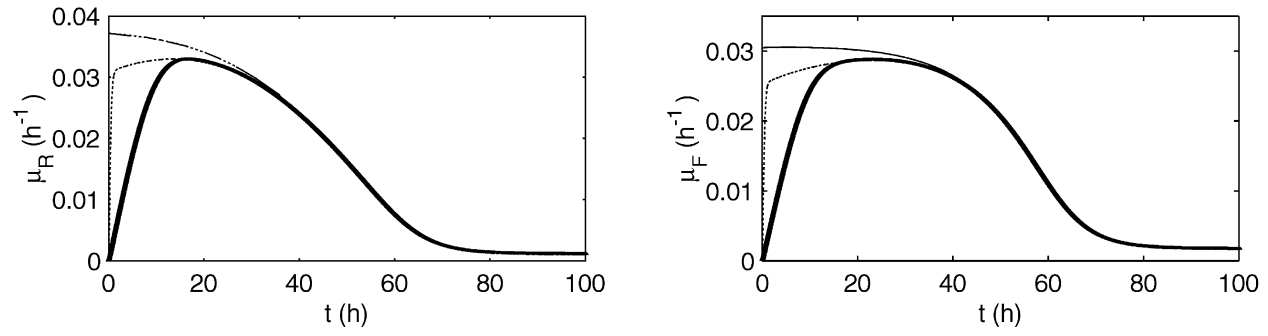

Fig. 4. Comparison of our design (- - ) with the classical one (. - ) for $\omega_{i}=10$.

illustrates the performance of the estimator with a square wave of the maximum specific growth rates $\mu_{\max , 1}(0.055 \rightarrow 0.155$ at time $t=20,40,60$, and 80$)$ and $\mu_{\max , 2}(0.045 \rightarrow 0.145$ at time $t=30,50,70$ and 100) and $\omega_{1}$ and $\omega_{2}$ equal to $10 \mathrm{~h}^{-1}$ : note that, although $X$ is increasing throughout the process operation of more than one order of magnitude $(0.18 \rightarrow 6)$, the convergence rate remains the same and is not affected by the value of $X$.

\subsection{Experimental results}

The observer-based estimator (52), (52), (63), (64) has been implemented on a $22 \mathrm{~L}$ pilot-scale bioreactor (see Siegwart et al., 1999). Fig. 6 presents one set of experimental results. The glucose (Fig. 6a) and lactate (Fig. 6b) are measured via a FIA biosensor device. The temper- ature and $\mathrm{pH}$ are maintained at constant values of 37 $\mathrm{C}$ and 7.2, respectively. The initial conditions were equal to those used in the numerical simulations. During this experiment, glucose was controlled via an adaptive linearizing controller to a low value equal to $1 \mathrm{mM}$. The observer-based estimator has been initialized as follows:

$\hat{\mu}_{R}(0)=\hat{\mu}_{F}(0)=0.01 \mathrm{~h}^{-1}, \quad \omega_{1}=\omega_{2}=0.325 \mathrm{~h}^{-1}$.

Note that in this particular instance, the same initial conditions and design parameter values have been able to give satisfactory results for both estimators. Because of the lack of reliable specific growth rate models, the validation of the estimation is performed by biomass concentration data: Fig. 6e compares the off-line data of biomass (o) with the estimation $X_{v}$ (dotted line) of biomass based on the mass balance equation in model (5), (6) and recontructed from the on-line estimates of the 

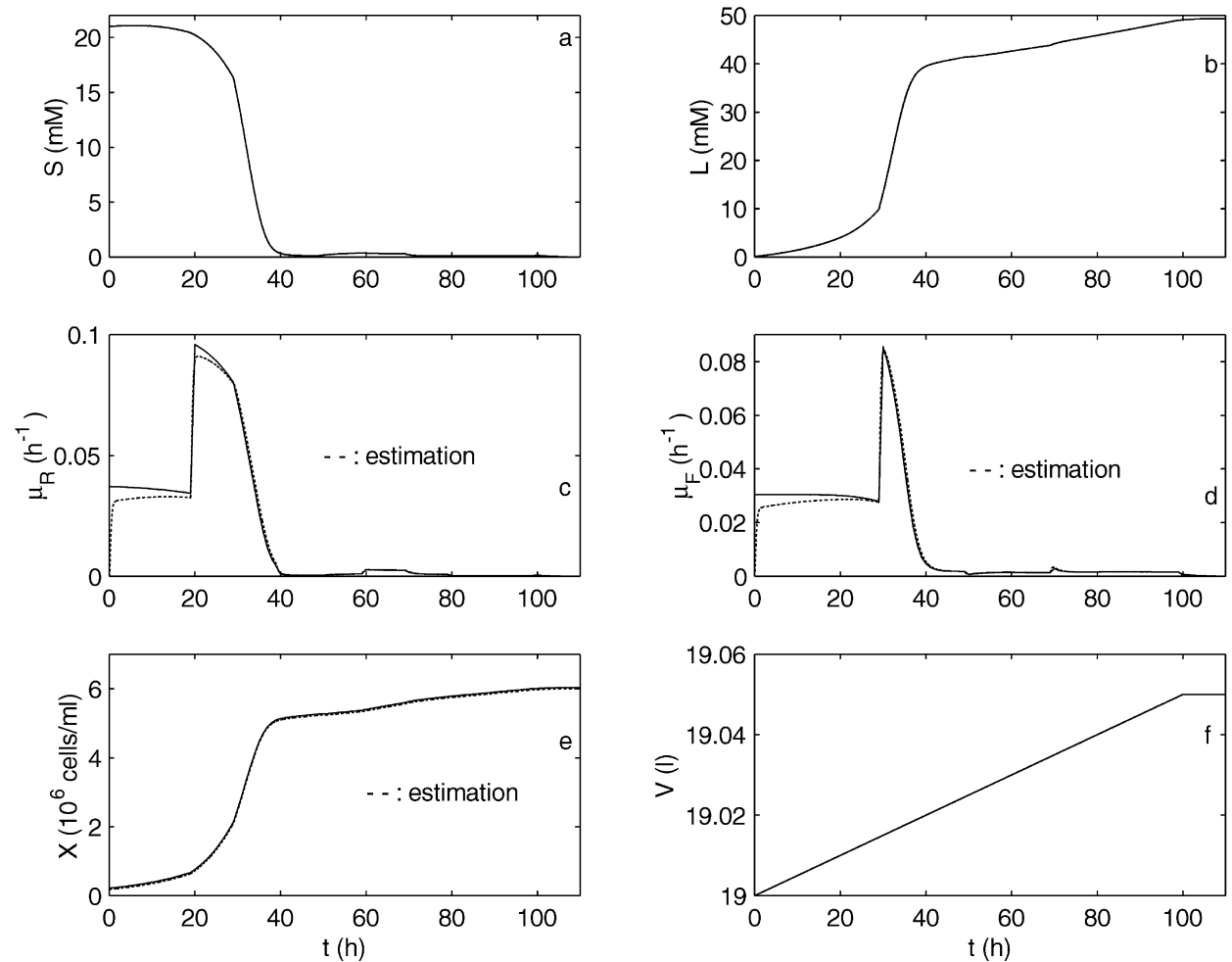

Fig. 5. Numerical simulation of the observer-based estimator with square wave variation of the maximum specific growth rates.
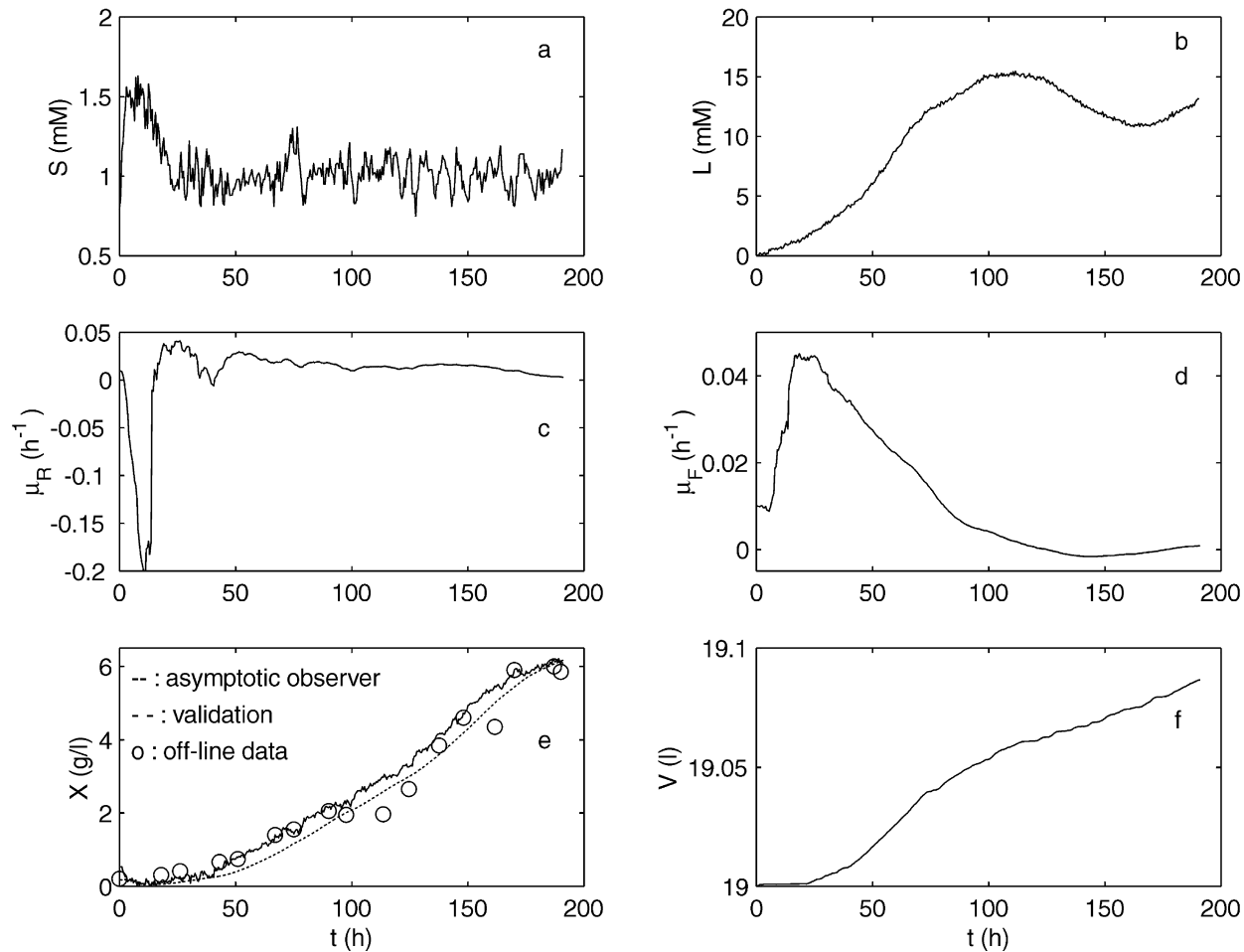

Fig. 6. Observer-based estimator: experimental results on an animal cell culture. 

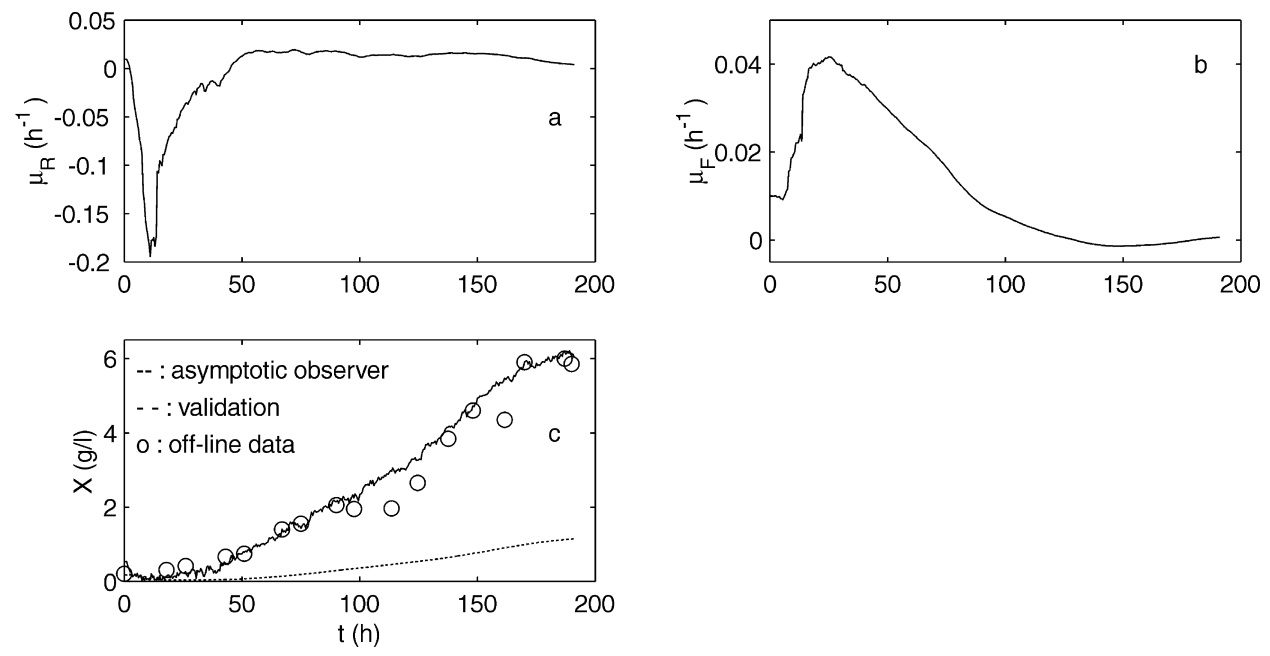

Fig. 7. Experimental results with non-optimal design parameters.

specific growth rates $\hat{\mu}_{R}$ and $\hat{\mu}_{F}$ :

$\frac{\mathrm{d} X_{v}}{\mathrm{~d} t}=\hat{\mu}_{R} X_{v}+\hat{\mu}_{F} X_{v}-D X_{v}$.

Note that the observer-based estimator detects the decrease of lactate around time $=120 \mathrm{~h}$ and gives a negative value to $\hat{\mu}_{F}$. Note also that the estimation of $\mu_{R}$ is pushed to negative values at the beginning of the culture largely due to the measurement noise.

The selection of the design parameters values has typically followed the procedure described in Section 5.3. Numerical simulations have been used to give the first initial guesses based on the plausible measurement noise and specific growth rate model, then they have been adjusted in order to obtain the best validation possible with the biomass experimental data.

It is worth noting that the sensitivity in the calibration procedure can largely depend on one application to another. Experience shows that the hydraulics play a key role in this sensitivity. Typically, the calibration is easy and the design parameters are very insentitive for processes where the hydrodynamics is large compared to the kinetics, i.e. for fedbatch reactors with large influent flow rate (and therefore large volume variations) (typical examples are the baker's yeast applications presented in Claes and Van Impe (1998), Pomerleau and Perrier (1990) and Pomerleau and Viel (1992)) and for continuous reactors. The calibration will be more time consuming, mainly because of the high sensitivity of the estimation results with respect to the design parameter values, in batch processes or in fedbatch reactors with low influent flow rate. The example is particularly interesting because it belongs to this class of processes. As a matter of illustration, Fig. 7 shows the estimation results when $\omega_{i}(i=1,2)$ is equal to 0.25 (i.e. $23 \%$ variation from the best ones): note that the estimated specific growth rates are smoother and that a good validation of the biomass concentration is not capable of being generated with this set of design parameter values.

\section{Conclusions}

This paper has been dedicated to the tuning of observer-based estimators. These type of estimators are an alternative to classical estimators like extended Kalman filters that exhibit limitations when applied to systems like chemical and biochemical processes. As indicated by their name, they have the basic structure of observers. The observer-based estimator presents the following specific characteristics: it does not need to introduce a black-box (nonlinear) modelling of the uncertain parameters, and the stability analysis does not lead to conservative tuning rules (as may be the case with $H_{\infty}$ based algorithms). These characteristics are advantages in many (bio)process applications. But they may also be limitations in other instances. In this respect, hybrid modelling (which combines mass and energy balance models with neural networks for the kinetics) opens an interesting avenue (e.g. Feyo de Azevedo, Dahm \& Oliveira, 1997).

A systematic tuning approach of the observer-based estimator, which allows a decoupled estimation of each parameter and the assignement of the estimator dynamics independently of the process dynamics has been proposed. The transformation that allows the decoupling is indeed a Lyapunov transformation. The tuning only requires choosing one (possibly two) design parameter(s) per estimated parameter. The performance of the proposed approach has been illustrated both in numerical simulation and with real-life data via the practical example of an animal cell culture. 


\section{Acknowledgements}

This paper presents the research results of the Belgian Programme on Inter-University Poles of Attraction initiated by the Belgian State, Prime Minister's office for Science, Technology and Culture. The scientific responsibility rests with its authors.

\section{References}

Atroune, D., Cheruy, A., Flandrois, J. P., \& Carret, G. (1988). Choice of the specific growth rate formulation in biotechnological processes. Proceedings of IMACS 12th world congress on scientific computation.

Bastin, G., \& Dochain, D. (1986). On-line estimation of microbial growth rates. Automatica, 22(6), 705-711.

Bastin, G., \& Dochain, D. (1990). On-line estimation and adaptive control of bioreactors. Amsterdam: Elsevier.

Bastin, G., \& Levine, J. (1990). On state reachability of reaction systems. Proceedings of 29th CDC (pp. 2819-2824).

Bourrel, S., Dochain, D., Babary, J. P., \& Queinnec, I. (1999). Modelling, identification and control of a denitrifying biofilter. Journal of Process Control, 10, 73-91.

Caminal, G., Lafuente, F. J., Lopez-Santin, J., Poch, M., \& Sola, C. (1987). Application of the extended Kalman filter to identification of enzymatic deactivation. Biotechnology and Bioengineering, 24, 66-369.

Claes, J. E., \& Van Impe, J. (1998). On-line monitoring and optimal adaptive control of the fed-batch baker's yeast fermentation. Proceedings of CAB7 7th International conference on computer applications in biotechnology (pp. 405-410).

Cannon, M., \& Slotine, J. -J. E. (1995). Space frequency localized basis functions networks for nonlinear system estimation and control. Neurocomputing, 9, 293-342.

Dochain, D. (1994). Design of linearizing adaptive controllers for nonisothermal reactors. International Journal of Control, 59(3), 689-710.

Dochain, D., \& Chen, L. (1992). Local observability and controllability of stirred tank reactors. Journal of Process Control, 2(3), 139-144.

Dochain, D., Perrier, M., \& Ydstie, B. E. (1992). Asymptotic observers for stirred tank reactors. Chemical Engineering Science, 47, 4167-4178.

Doyle III, F. J. (1997). Nonlinear inferential control for process applications. Proceedings of ADCHEM'97 (pp. 170-181).

Feyo de Azevedo, S., Dahm, B., \& Oliveira, F. R. (1997). Hybrid modelling of biochemical processes: A comparison with the conventional approach. Computers and Chemical Engineering, (Suppl.), 21, S751-756

Flaus, J. M., Pons, M. N., Cheruy, A., \& Engasser, J. M. (1989). Adaptive algorithm for estimation and control of fed-batch bioprocesses. In: M. N. Fish, R. I. Fox \& N. F. Thornhill, Proceedings of Computer Applications in Fermentation Technology: Modelling and control of biotechnology processes. (pp. 349-353) Oxford, Pergamon Press.

Goodwin, G. C., Mc Innis, B. C., \& Long, R. S. (1980). Adaptive control algorithms for waste water treatment and $\mathrm{pH}$ neutralization. Optimal Control Applications Methods, 3, 443-459.
Goodwin, G. C., \& Payne, R. L. (1977). Dynamic system identification: Experiment design and data analysis. New York: Academic Press.

Kiparissides, C., Mac Gregor, J. F., \& Hamielec, A. E. (1981). Suboptimal stochastic control of a continuous latex reactor. A.I.Ch.E. Journal, 27, 13-19.

Kwakernaak, H., \& Sivan, R. (1972). Linear optimal control systems. New York: Wiley.

Ljung, L. (1979). Asymptotic behavior of the extended Kalman filter as a parameter estimator for linear systems. IEEE Transactions on Automatic Control, 24, 36-50.

Mbihi, J., Dochain, D., \& Turgeon, A. (1993). Modelling of an autonomous power system at variable speed for transient simulation using Lagrange's laws. Canadian Electronics and Computer Engineering Journal, 18(3), 127-131.

MacGregor, J. F., Kojub, D. J., Penlidis, A., \& Hamielec, A. E. (1986). State estimation for polymerization reactors. Proceedings of IFAC Symposium on DYCORD '86 (pp. 147-152).

Oliveira, R., Ferreira, E. C., Oliveira, F., \& Feyo de Azevedo, S. (1996). A study on the convergence of observer-based kinetics estimators in stirred tank bioreactors. Journal of Process Control, 6(6), 367-371.

Ortega, R., \& Espinosa, G. (1993). Torque regulation of induction motors. Automatica, 29(3), 621-633.

Perrier, M., \& Dochain, D. (1993). Evaluation of control strategies for anaerobic digestion processes. International Journal of Adaptive Control Signal Processing, 7(4), 309-321.

Pomerleau, Y., \& Perrier, M. (1990). Estimation of multiple specific growth rates in bioprocesses. A.I.Ch.E Journal, 27, 231-236.

Pomerleau, Y., Perrier, M., \& Bourque, D. (1995). Dynamics and control of the fed-batch production of poly- $\beta$-hydroxybutyrate by methylobacterium extorquens. Proceedings of sixth international conference on computer applications in Biotechnology (pp. 107-112).

Pomerleau, Y., \& Viel, G. (1992). Industrial application of adaptive nonlinear control for baker's yeast production. In: N. M. Karim, $\&$ G. Stephanopoulos, Proceedings of fifth international conference on computer applications in biotechnology. (pp. 315-318) Oxford: Pergamon Press.

Reza Moheimani, S. O., Satkin, A. V., \& Petersen, I. R. (1996). Robust filtering, prediction, smoothing and observability of uncertain systems. Proceedings of 35th CDC (pp. 4794-4799).

Siegwart, P., Male, K., Côté, J., Luong, J. H. T., Perrier, M., \& Kamen, A. (1999). Adaptive control at low-level glucose concentration to study HEK-293 cell metabolism in serum pure cultures. Biotechnology Progress 15, 608-616.

Stephanopoulos, G., \& San, K. Y. (1984). Studies on on-line bioreactor identification. Biotechnology and Bioengineering, 26, 1176-1188.

Sulmon, D. (1997). Design and implementation of monitoring tools for a biotechnological process. Engineering thesis, Erasmus project, Université Catholique de Louvain, Belgium \& Universitat Stuttgart, Germany.

Tsobanakis, P., Lee, S. H., Phillips, J. A., \& Georgakis, C. (1992). Issues in the optimization, estimation and control of fed-batch bioreactors using tendency models. In: N.M. Karim, G. Stephanopoulos, Proceedings of the fifth international conference on computer applications in Biotechnology (pp. 71-76). Oxford: Pergamon Press.

Wells, C. H. (1971). Application of modern estimation and identification techniques to chemical processes. AIChE Journal, 17, 966-973. 